Editorial

\title{
Formation and Fluxes of Soil Trace Gases
}

\author{
Laura K. Meredith ${ }^{1, *(\mathbb{C})}$, Kristin Boye ${ }^{2,3}\left(\mathbb{D}\right.$, Kathleen Savage ${ }^{4}$ and Rodrigo Vargas ${ }^{5}(\mathbb{C}$ \\ School of Natural Resources and the Environment, University of Arizona, Tucson, AZ 85721, USA \\ Department of Earth System Science, Stanford University, Stanford, CA 94305, USA; kboye@stanford.edu \\ SLAC National Laboratory, Stanford Synchrotron Radiation Lightsource, Menlo Park, CA 94025, USA \\ Woods Hole Research Center, 149 Woods Hole Rd, Falmouth, MA 02540, USA; savage@whrc.org \\ Department of Plant \& Soil Science, University of Delaware, Newark, DE 19716, USA; rvargas@udel.edu \\ * Correspondence: laurameredith@arizona.edu; Tel.: +1-520-626-4213
}

Received: 2 April 2020; Accepted: 3 April 2020; Published: 16 April 2020

\section{Introduction}

Trace gas cycling is an important feature of the soil system. Current research is advancing knowledge on the formation and fluxes of soil trace gases through interdisciplinary approaches and the availability of new technological advances. In this Special Issue, research teams use novel methods to address challenges in soil system research, including how to (1) measure and scale processes with high temporal and spatial variability; (2) partition the net soil exchange of trace gases to independently quantify and study underlying biogeochemical processes; and (3) reconcile insights derived from fieldand laboratory-based investigations. Here, we summarize approaches in this research collection that fill specific knowledge gaps in trace gas biogeochemistry and address fundamental challenges in soil system science.

\section{Background}

Accurately modeling and predicting the fate of different trace gases requires a deep understanding of the major components of their biogeochemical cycles. Ultimately, this knowledge is translated into mathematical equations to represent biophysical dynamics at local, regional, or global scales. Trace gas emissions and uptake from soil are often under-recognized in global element cycles (e.g., carbon (C), nitrogen $(\mathrm{N})$ ), and in many models these emissions are represented using a residual approach in an effort to close the mass balance. Where explicitly considered, underlying drivers and feedback loops are often not well developed or understood. For example, soils are key contributors to global emissions of greenhouse gases such as methane $\left(\mathrm{CH}_{4}\right)$ and nitrous oxide $\left(\mathrm{N}_{2} \mathrm{O}\right)$, but the complexity of biological and abiotic soil processes that promote or limit emissions is still not well understood. Even the explicit inclusion of heterotopic respiration (i.e., soil $\mathrm{CO}_{2}$ fluxes derived from decomposition of organic matter) is still a matter of debate for earth system models' benchmarking and parametrization. Furthermore, soils are emitters of less-studied trace gases including volatile metal(loid)s (e.g., methylated arsenic and mercury), and the regulating mechanisms of fluxes that constitute important export pathways for these elements are still unclear. Consequently, there is a need to identify the main biogeochemical drivers of trace gases to properly represent them in process-based models.

We propose that improving understanding of trace gas formation and fluxes in soil systems requires addressing three grand challenges related to soil biogeochemical research: (a) soil processes have high spatial and temporal variability; (b) soils support simultaneous production and consumption of the same trace gas; and (c) and laboratory-and field-based insights often do not agree.

Soil processes are dynamic in space and time, making it important to capture locations and time points that support disproportionate activity-so-called "hot spots" and "hot moments". Traditionally, technical and cost limitations have been prohibitive to capturing the data required to accurately 
constrain soil dynamics and their cumulative effects, from the ecosystem scale to the global scale. Here, we highlight how recent advances in technology and associated analysis frameworks may help constrain soil gas formation and fluxes and facilitate their robust integration into global cycles.

Measurements of the net soil exchange of trace gases provide insights into biological and abiotic processes in soils. Many production and consumption processes affect trace gases in soils (e.g., volatilization/condensation, photo- and thermo-chemical reactions, physical (de)sorption, microbe-mediated conversions), and in most cases more than one process affects soil fluxes of a given trace gas. To disentangle simultaneous trace gas production and/or consumption processes (i.e., cryptic cycles), methods are needed that partition net soil exchange (NSE) measurements into gross flux components. Partitioning NSE is important to correctly quantify and attribute individual gas formation and flux processes and build representative models for their study. Here, we summarize research approaches to partition NSE, including process suppression, process sensitivity, and stable isotope analysis.

An important approach to close knowledge gaps on soil gas fluxes and their underlying drivers is to conduct laboratory experiments. These approaches are ideal for testing targeted hypotheses under controlled conditions and to examine specific factors, thresholds, and tipping points regulating gas formation and fluxes when compared to field investigations (which are influenced by multiple confounding effects). However, laboratory experiments are inherently associated with compromises and detachment from the complexity of the natural environment. In the case of gas formation and fluxes, this is particularly evident with the removal of natural weather fluctuations that directly and rapidly influence the rate and direction of gas exchange among soil, plants, and the atmosphere. Further, laboratory studies will never fully capture the spatial heterogeneity across multiple scales or the temporal variability that multiple contributions in this Special Issue show have a large impact on soil trace gas processes. However, with careful and insightful design and targeted aims, laboratory experiments can provide highly valuable information for understanding in detail the biogeochemical processes controlling trace gas dynamics.

In this editorial, we synthesize current methods for scaling spatial and temporal variability, partitioning trace gas fluxes for process-based studies, and reconciling field and lab studies, using examples from this Special Issue.

\section{Advancing Knowledge of the Formation and Fluxes of Soil Gases}

\subsection{Scaling Spatial and Temporal Variability}

Recent advances in technology have allowed broad deployment of measurement systems that capture high-temporal-frequency gas-phase measurements of important trace elements (e.g., $\mathrm{C}$ and $\mathrm{N})$ both above and below ground, thus constraining responses to drivers at relevant temporal and spatial scales. Increased computational power now permits investigating "big data" streams using novel data-fusion modeling techniques that further understanding of process controls and responses across time and space.

Vargas et al. (2018) used an integrated data and model approach to understand the temporal, pulsed behavior of soil carbon dioxide $\left(\mathrm{CO}_{2}\right)$ fluxes, illustrating the dynamic and nonlinear nature of soil processes linked to trace gas cycling [1]. Using high-temporal-frequency measurements of $\mathrm{CO}_{2}$ fluxes to capture transient responses (i.e., "hot moments") of $\mathrm{CO}_{2}$ release in response to precipitation events, they applied novel timeseries and modeling approaches to understand responses. This work outlines a framework for measuring and modeling transient responses in $\mathrm{CO}_{2}$ that can be extended to other trace gases exhibiting "hot moment" dynamics, such as $\mathrm{CH}_{4}$ and $\mathrm{N}_{2} \mathrm{O}$.

Constraining temporal soil gas dynamics has been limited by sampling techniques and tools, especially traditional manual sampling. In particular, it has been challenging to partition co-occurring processes within the soil column (Section 3.2), e.g., the production and consumption (via oxidation) of $\mathrm{CH}_{4}$, from measurements that poorly resolve temporal variations in these processes. Leveraging 
the increasing availability of automated, sub-hourly soil flux measurement systems, Nielson et al. (2018) illustrate a novel use of a relatively new chamber-based measurement system for stable $C$ isotopes to distinguish in-situ, co-occurring fluxes in methane cycling [2]. Specifically, from continuous, high-temporal-frequency flux measurements of the $13-\mathrm{C}$ stable isotope composition $\left(\delta^{13} \mathrm{C}\right)$ of $\mathrm{CO}_{2}$, they partition soil $\mathrm{CH}_{4}$ oxidation rates by determining the contribution of this process to heterotrophic respiration. These high-frequency measurements reveal previously uncharacterized diel patterns in $\mathrm{CH}_{4}$ oxidation in peatlands, peaking during the day and falling to negligible levels at night.

Roby et al. (2019) integrate studies that constrain both the spatial and temporal variability of soil $\mathrm{CO}_{2}$ fluxes and compare the influence of vegetation cover, illustrating the importance of plants' inputs and interactions with soil systems [3]. Specifically, high-temporal-frequency chamber-based soil and eddy covariance flux tower $C$ fluxes were compared across three semi-arid sites with similar climatic forcing but differing vegetation structures. They employed the trenching method to exclude vegetation influence, to further partition the effects of drivers on autotrophic and heterotrophic respiration components. By assessing spatial patterns with high-frequency data, Roby et al. (2019) showed that vegetation structure plays a significant role over integrated time scales in controlling spatial patterns of soil C flux in a semi-arid environment and suggest that vegetation regulation of water plays a role in the temporal patterns of soil carbon flux.

Jones et al. (2018) highlight the spatial variability in soil $\mathrm{CH}_{4}$ emissions and illustrate the power of high coverage of measurements (here, spatially) and the potential to infer temporal dynamics from spatial data (space-for-time substitution) [4]. The study was conducted in a wetland in the Peruvian Andes with soils ranging from freely draining upland to inundated wetland. The drainage distribution provided the opportunity to examine micro-topological control of $\mathrm{CH}_{4}$ fluxes. Combining long-term and intensive sampling strategies, Jones et al. (2018) found considerable variation in $\mathrm{CH}_{4}$ fluxes, primarily driven by topography stemming from patterns in soil $\mathrm{O}_{2}$ and its influence on the balance of $\mathrm{CH}_{4}$ production and consumption. They further report net $\mathrm{CH}_{4}$ emissions in well-drained ridge soils, indicating that precipitation patterns influence the $\mathrm{CH}_{4}$ NSE balance at the micro scale, changing sites that would be presumed net $\mathrm{CH}_{4}$ sinks to transient sources. Together, landscape topography and precipitation control spatial and temporal patterns of soil $\mathrm{O}_{2}$, making this ecosystem a net source in regional budgets.

High spatial variability in gas fluxes illustrates the need for systematic surveys of soil gas flux potential. Often, estimates of the magnitude of soil gas fluxes are based on small datasets restricted to a small set of biomes or land use types. These data sets, though limited and with poorly defined variability, can propagate into global atmospheric budgets and models. This requires systematic surveys of soil fluxes over larger ranges of biomes and soil properties, as is illustrated in this issue by Meredith et al. (2018) and Kaisermann et al. (2018) for soil carbonyl sulfide (OCS) fluxes [5,6].

\subsection{Partitioning Fluxes for Process-Based Studies}

The net soil-atmosphere flux of trace gases commonly contains two or more gross production and/or consumption processes. In fact, this is so widespread for trace gases including $\mathrm{CO}_{2}$ that Cueva et al. (2019) argue that, rather than assuming any soil flux is the result of a unidirectional process, the expectation that multiple processes combine into a net soil exchange (NSE) - akin to the concept of net ecosystem exchange (NEE) — should be the primary hypothesis for soils [7]. Contributions in this Special Issue partition fluxes for process-based investigation using approaches that include: (1) modifying soil conditions to isolate one process over others; (2) considering the specific sensitivity of a single process; and (3) using stable isotope techniques such as natural abundance and pulse labeling approaches. Developing methods to partition NSE into contributions of individual processes is important for quantifying the contributions of various processes to the net soil flux, attribution of the process(es) driving changes to net soil flux, and developing models that encode trace gas producing and consuming processes and their individual drivers for more accurate predictions. 
One approach for partitioning NSE into individual components is to alter the soil system in ways that isolate one process while suppressing other process(es). Meredith et al. (2018) investigated the drivers of abiotic emissions of OCS, an important carbon dioxide analogue, from soils, by air-drying soils to suppress microbial activity-the competing microbial OCS consumption process [5]. In the field, Roby et al. (2019) used trenching manipulations to exclude the contributions of roots from trenched plots and partition the contributions of heterotrophic (microbial decomposition) and autotrophic (root-associated) respiration to the total $\mathrm{CO}_{2}$ efflux [3]. Vargas et al. (2018) and Roby et al. (2019) both extend this concept to the natural patchiness of the semi-arid landscape, which supports patches of relatively little (bare soil) or dense (near grass) root density [1,3]. Suppressing soil processes may directly or indirectly affect the process of interest, as noted by Miller et al. (2019) for inhibitors of methanogenesis [8], consistent with observations by Jones et al. (2018) of co-varying gross trace gas fluxes (e.g., $\mathrm{CH}_{4}$ consumption and production) [4], which must be considered when applying this method.

A second approach leverages the specific sensitivity of processes to drivers and uses process-driver relationships to identify the presence of multiple processes, constrain process rates, and evaluate model sensitivity to drivers of NSE. Evaluating NSE against potential driving variables can reveal the presence of multiple processes. For example, in an experimental basaltic soil system, Cueva et al. (2019) observed strong negative NSE (e.g., net $\mathrm{CO}_{2}$ soil sink) driven by weathering reactions [7]. By evaluating the relationship between NSE and soil diffusivity and water potential, they found that, while drier soils facilitate diffusion of $\mathrm{CO}_{2}$ into soils for weathering uptake, the trend was reversed at intermediate soil moisture potentials that can support microbial activity in soils, suggesting the presence of a second $\mathrm{CO}_{2}$ flux (i.e., production) such as microbial respiration. Additionally, the nature of a process may be clarified from relationships with drivers. For example, Meredith et al. (2018) observed an exponential increase in OCS production in dry soils without a decline at high temperatures that would denature enzymes, suggesting the process is driven by zero-order chemical production and not biological production [5]. First-order trace gas consumption processes are sensitive to trace gas (substrate) concentrations, which Kaisermann et al. (2018) leverage to partition OCS NSE by varying OCS concentrations to stimulate microbial consumption rates, while OCS production does not vary [6]. They derive gross consumption and production rates from this method and additionally use reactive trace gas models to account for physical factors of the soil system and derive underlying bulk enzymatic rates for OCS consumption. Roby et al. (2019) integrate data with simple models to identify periods and conditions under which the processes of heterotrophic and autotrophic respiration separately contribute to NSE [3]. These insights can also be derived from emerging statistical approaches such as partial wavelet coherence analysis, applied by Vargas et al. (2018), which reveal the sensitivity of different components of NSE to environmental drivers [1]. Finally, research in soil formation and fluxes also faces challenges in partitioning net fluxes at the ecosystem scale (NEE), especially when traditional soil flux measurement methods are not possible or cause significant artifacts, as is the case for the NSE of ammonia $\left(\mathrm{NH}_{3(\mathrm{~g})}+\mathrm{NH}_{4}{ }^{+}\right.$(s,l,particleg) $)$. Hrdina et al. (2019) measure the NEE of ammonia and use compensation point models parameterized with soil and canopy $\mathrm{N}$ measurements to partition NEE and isolate NSE from canopy fluxes [9], an important scientific advancement for relatively understudied trace gases like ammonia.

Finally, stable isotopes are powerful tools for partitioning NSE by either natural abundance or pulse labeling approaches. Natural abundance stable isotope approaches measure unaltered stable isotope ratios to attribute fluxes to particular pools and quantify process rates. For example, Nielson et al. (2018) constrain rates of aerobic $\mathrm{CH}_{4}$ consumption (via) oxidation in northern peatlands using natural abundance of stable $\mathrm{C}$ isotopes $\left(\delta^{13} \mathrm{C}\right)$ of $\mathrm{CO}_{2}$ to distinguish in-situ, co-occurring fluxes in methane cycling [2]. The approach characterizes key factors in the laboratory (fractionation factor, stable isotope profiles of SOM and pore water) to use $\mathrm{CO}_{2}$ and $\mathrm{CH}_{4}$ bulk and isotope fluxes and a two-source isotope mixing model to deduce the potential contribution of $\mathrm{CH}_{4}$ oxidation to in-situ fluxes over a 10-day period. This method allows partitioning of cryptic $\mathrm{CH}_{4}$ cycling (gross oxidation and methanogenesis 
fluxes) from NSE and also evaluates the relative importance of contributions of oxidation products (here $\mathrm{CO}_{2}$ ) to the total soil $\mathrm{CO}_{2}$ efflux [2]. In contrast, isotope labeling approaches artificially change the stable isotope ratio of the soil gas or its production source, but usually without significantly altering the bulk concentration, and track the labeled compound through soil gas producing and consuming fluxes and pools. For example, Miller et al. (2018) used the stable isotope labeling approach to constrain anaerobic methane oxidation. They isotopically enriched soil cores with ${ }^{13} \mathrm{CH}_{4}$ in anaerobic laboratory incubations to quantify anaerobic methane oxidation from measurements of ${ }^{13} \mathrm{CO}_{2}$ production and quantify this cryptic carbon cycling process alongside $\mathrm{CO}_{2}$ production from soil organic matter respiration and $\mathrm{CH}_{4}$ production by methanogenesis [8]. Jones et al. (2018) constrained aerobic methane oxidation and partitioned net $\mathrm{CH}_{4}$ fluxes through ${ }^{13} \mathrm{CH}_{4}$ isotope enrichment in aerobic laboratory incubations [4]. In both cases, isotope mixing models are used to interpret measurements. Stable isotope approaches often require assumption and/or measurement of various factors (e.g., fractionation factors, substrate isotope ratio), which may be more feasible to measure in the laboratory where isotope labeling typically occurs $[4,8]$, but are likely impractical to measure at the same temporal resolution as in-situ natural abundance isotope studies (e.g., [2]). Taken together, these isotope approaches reveal otherwise-hidden contributions of various biogeochemical processes to the overall soil flux and advance understanding of soil gas formation and fluxes.

\subsection{Reconciling Field and Lab Studies}

Multiple challenges exist in reconciling gas flux estimates and measurements based on laboratory investigations relative to field measurements, as shown by Fidel et al. (2019) in this Special Issue [10]. Although the laboratory incubations they performed were made with soil from the same field plots and the same type of biochar as the field experiment, they were unable to obtain consistency between biochar effects on $\mathrm{N}_{2} \mathrm{O}$ and $\mathrm{CO}_{2}$ emissions as measured in the lab relative to the field. Along these same lines, Sapkopta et al. (2020) review the available literature to understand how reductions in irrigation in agricultural systems influence soil emissions of $\mathrm{CO}_{2}, \mathrm{~N}_{2} \mathrm{O}$, and $\mathrm{CH}_{4}$ and their associated global warming potential, and find consistent increases in $\mathrm{CO}_{2}$ and decreases in $\mathrm{CH}_{4}$ emissions, but inconsistent trends in the response of soil $\mathrm{N}_{2} \mathrm{O}$ emissions to irrigation treatment [11]. In both manuscripts, reasons for discrepancy between laboratory and field and across field studies are discussed, some of which may be relatively easily addressed in future studies, through even more strictly regimented matching of experimental materials and designs and more expansive field experimental approaches [10,11]. That said, laboratory experiments will always have challenges to represent natural temperature and soil moisture fluctuations, plant and other biological inputs/effects, and differences in physical and biochemical properties of soil (i.e., spatial heterogeneity). As a consequence, the design of laboratory experiments requires recognition of factors that are likely to influence the examined trace gas fluxes and formation processes, but that are not feasible to account for or mimic in a laboratory setting. An approach to reconciling this discrepancy must, then, be developed, such that the results of the laboratory study provides information that is meaningful and complementary to what can be achieved through field investigations. For example, Meredith et al. (2018) and Kaisermann et al. (2018) both utilized the benefits of a controlled laboratory environment and access to advanced analytical equipment to test specific hypotheses related to the spatial variability observed between different soil types and land uses for the soil production and uptake of OCS. Instead of focusing on obtaining the most environmentally realistic and directly scalable measurements of OCS production and uptake rates possible, these two studies aimed to elucidate factors (e.g., nitrogen fertilization/availability, soil carbon, and organic sulfur species) that were hypothesized as potential drivers behind variability in OCS fluxes in previous studies. Similarly, Jones et al. (2018) utilized laboratory incubations, allowing for isotopic labeling and controlled environmental conditions, in parallel with their elaborate field measurements, to assess gross fluxes of $\mathrm{CH}_{4}$ and further elucidate underlying mechanisms that could explain their observed field variability in $\mathrm{CH}_{4}$ emissions. This approach may be well suited to more conclusively pinpoint impacts on soil GHG emissions by reducing irrigation to agricultural systems [11]. 
Another example of the utility of laboratory studies for understanding natural trace gas fluxes and processes is provided by Miller et al. (2019), who used intact soil cores brought back to the laboratory from Alaskan peatlands to reveal the important role of anaerobic $\mathrm{CH}_{4}$-oxidizing microbes for net $\mathrm{CH}_{4}$ fluxes in these environments. Incubating intact soils helps preserve some realistic conditions, while increasing controllability. Although the exact controls and quantitative contributions from this process will require further investigation, Miller et al. (2019) provide convincing evidence that anaerobic $\mathrm{CH}_{4}$ oxidation cannot be neglected as a consumption process in arctic peatlands, and as such, pinpoint the necessity of laboratory studies to fully understand the drivers and partitioning mechanisms behind trace gas formation and fluxes within soils and between soil and atmosphere.

Finally, Cueva et al. (2019) exemplifies a study that falls somewhere between laboratory and field-scale investigations in their utilization of the Biosphere 2 facility to examine a previously neglected, or even deliberately disregarded, $\mathrm{CO}_{2}$ flux from the atmosphere to soils. Normally, the soil $\mathrm{CO}_{2}$ flux is regarded as unidirectional from soil to the atmosphere; i.e., soils are considered net sources of $\mathrm{CO}_{2}$, which Cueva et al. (2019) dispute with their study, highlighting the importance of carbonate weathering as a net sink for $\mathrm{CO}_{2}$ in arid and alkaline soils. Biosphere 2 is a (so-far) quite unique example of a facility where laboratory control and instrumentation opportunities are scaled to field settings, although still missing much of the spatial and weather-driven variability exhibited in natural field conditions. In spite of the limitations regarding selection of soils and environmental conditions represented within a facility like Biosphere 2, the types of studies (as exemplified by Cueva et al. (2019)) that they support represent very important links between the natural, heterogeneous environment (which often prevents complete partitioning and understanding of underlying mechanisms) and highly controlled laboratory studies of only one or a few presumed important processes and/or factors.

\section{Conclusions}

New technological developments including instrumentation and techniques have enhanced research on formation and fluxes of soil trace gases in the last decade. Research has moved from spot-specific single-gas measurements (e.g., for $\mathrm{CO}_{2}$ ) to multiple (e.g., $\mathrm{CO}_{2}$ and $\mathrm{CH}_{4}$ ) or novel trace gases (e.g., OCS) at unprecedented temporal resolutions (e.g., hourly measurements). Furthermore, new isotopic techniques are providing insights into previously unknown soil processes and are promising to address the emerging challenges of apparent "cryptic" cycles of soil trace gases. This Special Issue brings together novel research on formation and fluxes of soil trace gases across multiple ecosystems and highlights three main challenges for soil biogeochemical research.

First, now more than ever, the scientific community is aware that soil processes have high spatial and temporal variability, where "hot moments" and "hot spots" exist. These spatial and temporal high rates need to be better quantified, and the underlying biogeochemical drivers need to be identified to properly represent them in process-based models. Second, diffusion of soil trace gases has usually been assumed to be in a thermodynamic "steady state" for modeling purposes, but new research has shown that this assumption may not be valid, as soils support simultaneous production and consumption of the same trace gas that may vary along the soil profile. Finally, there is a critical need to reconcile and complement laboratory- and field-based measurements and interpretations to develop unified and strong theories about soil trace gas dynamics. We hope that this Special Issue motivates a better synergy between laboratory experiments, field measurements and modelers to advance our understanding of soil processes. The ultimate goal of using/developing new technologies and collaborating across disciplines may be to improve our prognostic capabilities for soil processes under different environmental scenarios to address ecological grand challenges. Are we ready?

Funding: L.K.M. was supported by United States Department of Energy (DOE) Small Business Innovative Research and Small Business Technology Transfer (DE-SC0018459). K.B. was supported by the US DOE Office of Science, Office of Biological and Environmental Research (BER), through the SLAC National Accelerator Laboratory scientific focus area (SFA) (Contract No. DE-AC02-76SF00515). R.V. was supported by the National Science Foundation (1652594). 
Acknowledgments: We thank all authors for their valuable collaboration and contributions to this Special Issue. The manuscripts included in this Special Issue represent the high-quality interdisciplinary research that is advancing understanding of trace gas cycling in soil systems. We are grateful to the international team of reviewers for their thoughtful and constructive critiques of the manuscripts assessed.

Conflicts of Interest: The authors declare no conflict of interest.

\section{References}

1. Vargas, R.; Sánchez-Cañete P., E.; Serrano-Ortiz, P.; Curiel Yuste, J.; Domingo, F.; López-Ballesteros, A.; Oyonarte, C. Hot-Moments of Soil $\mathrm{CO}_{2}$ Efflux in a Water-Limited Grassland. Soil Syst. 2018, 2, 47. [CrossRef]

2. Nielsen, C.S.; Hasselquist, N.J.; Nilsson, M.B.; Öquist, M.; Järveoja, J.; Peichl, M. A Novel Approach for High-Frequency in-situ Quantification of Methane Oxidation in Peatlands. Soil Syst. 2018, 3, 4. [CrossRef]

3. Roby, M.C.; Scott, R.L.; Barron-Gafford, G.A.; Hamerlynck, E.P.; Moore, D.J.P. Environmental and Vegetative Controls on Soil CO2 Efflux in Three Semiarid Ecosystems. Soil Syst. 2019, 3, 4. [CrossRef]

4. Jones, S.P.; Diem, T.; Arn Teh, Y.; Salinas, N.; Reay, D.S.; Meir, P. Methane Emissions from a Grassland-Wetland Complex in the Southern Peruvian Andes. Soil Syst. 2018, 3, 2. [CrossRef]

5. Meredith, L.K.; Boye, K.; Youngerman, C.; Whelan, M.; Ogée, J.; Sauze, J.; Wingate, L. Coupled Biological and Abiotic Mechanisms Driving Carbonyl Sulfide Production in Soils. Soil Syst. 2018, 2, 37. [CrossRef]

6. Kaisermann, A.; Jones, S.; Wohl, S.; Ogée, J.; Wingate, L. Nitrogen Fertilization Reduces the Capacity of Soils to Take up Atmospheric Carbonyl Sulphide. Soil Syst. 2018, 2, 62. [CrossRef]

7. Cueva, A.; Volkmann, T.H.M.; Haren, J.; van Troch, P.A.; Meredith, L.K. Reconciling Negative Soil $\mathrm{CO}_{2}$ Fluxes: Insights from a Large-Scale Experimental Hillslope. Soil Syst. 2019, 3, 10. [CrossRef]

8. Miller, K.; Lai, C.-T.; Dahlgren, R.; Lipson, D. Anaerobic Methane Oxidation in High-Arctic Alaskan Peatlands as a Significant Control on $\mathrm{Net}^{\mathrm{CH}_{4}}$ Fluxes. Soil Syst. 2019, 3, 7. [CrossRef]

9. Hrdina, A.; Moravek, A.; Schwartz-Narbonne, H.; Murphy, J. Summertime Soil-Atmosphere Ammonia Exchange in the Colorado Rocky Mountain Front Range Pine Forest. Soil Syst. 2019, 3, 15. [CrossRef]

10. Fidel, R.; Laird, D.; Parkin, T. Effect of Biochar on Soil Greenhouse Gas Emissions at the Laboratory and Field Scales. Soil Syst. 2019, 3, 8. [CrossRef]

11. Sapkota, A.; Haghverdi, A.; Avila, C.C.E.; Ying, S.C. Irrigation and Greenhouse Gas Emissions: A Review of Field-Based Studies. Soil Syst. 2020, 4, 20. [CrossRef]

(C) 2020 by the authors. Licensee MDPI, Basel, Switzerland. This article is an open access article distributed under the terms and conditions of the Creative Commons Attribution (CC BY) license (http://creativecommons.org/licenses/by/4.0/). 